\title{
Consumo de energía y nutrimentos en mujeres mexicanas en edad reproductiva*
}

Mario Flores, M.C., ${ }^{(1)}$ Hugo Melgar, M.C., Dr. Med., ${ }^{(1)}$ Cecilia Cortés, Lic. en N utr., (1) Marta Rivera, Lic. en N utr., ${ }^{(1)}$ Juan Rivera, M. Sc., Ph. D., ${ }^{(1)}$ Jaime Sepúlveda, M.C., M. Sc., Ph. D. ${ }^{(2)}$

\section{Flores M, Melgar H, Cortés C, Rivera M, Rivera J, Sepúlveda J. Consumo de energía y nutrimentos en mujeres mexicanas en edad reproductiva. Salud Publica Mex 1998;40:161-171.}

\section{Resumen}

Objetivo. Analizar el consumo de energía y nutrimentos de mujeres mexicanas de 12 a 49 años de edad. Material y métodos. La información dietética se obtuvo por recordatorio de 24 horas en 9101 mujeres participantes en la Encuesta N acional de Nutrición de 1988 y se contrastó con características sociodemográficas y estado fisiológico. El consumo de nutrimentos se comparó con las recomendaciones de ingestión dietética (RID). Resultados. La mediana del consumo energético fue de $1568 \mathrm{kcal} / \mathrm{día}$. La dieta estuvo conformada por $15 \%$ de proteína, $60 \%$ de hidratos de carbono y $25 \%$ de grasa. La proporción de mujeres con una ingestión inferior a la mitad de las RID fue de 70\% para vitamina $A, 75 \%$ para B6, $56 \%$ para vitamina C, $33 \%$ para B12,69\% para folato, $33 \%$ para calcio y $22 \%$ para hierro. Conclusiones. Los hallazgos muestran deficiencias importantes en la dieta, las cuales son más acentuadas en mujeres embarazadas o nodrizas, en las de menor nivel socioeconómico, en las que habitan en áreas predominantemente rurales 0 indígenas y en las de la región sur.

Palabras clave: micronutrientes; mujeres; nutrición de la madre; encuestas nutricionales; México
Flores M, Melgar H, Cortés C,

Rivera M, Rivera J, Sepúlveda J.

Energy and nutrient intake

in mexican women of reproductive age.

Salud Publica Mex 1998;40:161-171.

\begin{abstract}
A bstract
Objective.To analyze energy and nutrient consumption in Mexican women from 12 to 49 years of age. Material and methods. D ietetic information was gathered by a $24 \mathrm{~h}$ recall from 9101 women who participated in the $\mathrm{N}$ ational $\mathrm{N}$ utrition Survey conducted in 1988. These data were compared with sociodemographic and physiologic characteristics. N utrient consumption was compared with the Recommended Daily Allowances (RDA). Variance analysis and t-test were used to evaluate group differences. Results. Mean energy consumption was $1721 \mathrm{kcal}$ per day.The diet consisted of $15 \%$ protein, $60 \%$ carbohydrates and $25 \%$ fat. The proportion of women with dietary intakes lower than $50 \%$ of the RDA was $70 \%$ for vitamin A, $75 \%$ for vitamin B6, 56\% for vitamin C, $33 \%$ for vitamin B12, $69 \%$ for folate, $33 \%$ for calcium and $22 \%$ for iron. Conclusion. Results show important deficiencies in the diet, predominantly in pregnant and lactating women, in women from low socioeconomic levels and in rural and indigenous women of south of the Mexican republic.
\end{abstract}

Keywords: micronutrients; women; mother nutrition; nutritional surveys; Mexico

* La investigación fue financiada parcialmente por el Consejo N acional de Ciencia y Tecnología, México (proyecto num. 3902P-M9607).

(1) Dirección de Nutrición y Salud, Centro de Investigación en Salud Poblacional (CISP), Instituto N acional de Salud Pública (IN SP), México.

(2) Dirección General, IN SP, México.

Fecha de recibido: 30 de junio de 1997 - Fecha de aprobado: 13 de febrero de 1998

Solicitud de sobretiros: Dr. Mario Flores. Dirección de Nutrición y Salud, Centro de Investigación en Salud Poblacional, Instituto N acional de Salud Pública. Avenida Universidad 655, colonia Santa María A huacatitlán, 62508 Cuernavaca, Morelos, México. Correo electrónico: mflores@ insp3.insp.mx 
$\mathrm{L}$ as mujeres en edad reproductiva conforman un grupo cuyas funciones son de gran relevancia para la sociedad. El desempeño de dichas funciones depende en gran medida de su estado de salud y de nutrición. Por su función primordial en la reproducción y por su importante papel en la crianza de los hijos, la mujer en edad reproductiva es actor central en la producción y la formación de las generaciones futuras.

Durante el embarazo y la lactancia las necesidades nutricias de la mujer aumentan por las demandas que involucra la formación o la alimentación de un nuevo ser. ${ }^{1}$ Durante estas etapas la mujer está más expuesta al riesgo de sufrir deficiencias nutrimentales, especialmente si su estado preconcepcional es inadecuado. ${ }^{2}$ Además, por su rol de género, muchas mujeres en México enfrentan posiciones de subordinación y discriminación en términos de reconocimiento de sus necesidades, ${ }^{3}$ lo que, aunado a la creciente participación de la mujer en actividades productivas, impone demandas de energía y nutrimentos que, de no ser satisfechas, pueden llevar a deficiencias nutrimentales y deterioro de la salud. ${ }^{4}$

Existe evidencia de que el peso al nacer, así como la salud del recién nacido-los cuales tienen consecuencias definitivas en el crecimiento y el desarrollo-, dependen en gran medida del estado nutricional de la madre (medido por antropometría), no solamente durante sino mucho antes del embarazo. ${ }^{5,6}$ Asimismo, la calidad y la cantidad de la dieta de la mujer en la etapa prenatal tienen gran importancia para el producto del embarazo. $^{7-10}$

Algunas deficiencias específicas de micronutrimentos en la dieta pueden ser responsables de efectos nocivos tanto en la madre como en el producto del embarazo. Tal es el caso de la anemia por deficiencia de hierro, a la cual se ha responsabilizado de ser causa principal o contribuyente de 20 a $40 \%$ de las muertes maternas en países en desarrollo. ${ }^{11}$ Esta misma deficiencia se ha asociado con un mayor riesgo de muerte fetal, anomalías congénitas, prematurez y bajo peso al nacer, ${ }^{12}$ así como con una escasa producción de leche, menor duración de la lactancia y, por consiguiente, una edad de ablactación más temprana en mujeres primíparas..$^{13}$ Es sabido que la duración de la lactancia materna, así como el momento de introducción de alimentos complementarios y líquidos, determinan en gran medida el estado nutricional de los niños, los patrones de enfermedades infecciosas y la mortalidad infantil. ${ }^{14}$

Otras deficiencias de micronutrimentos, como las vitaminas A y E, el folato, la riboflavina y la niacina han sido a su vez relacionadas con efectos adversos en el producto del embarazo. ${ }^{15-19}$
Hasta antes de 1988, año en que se llevó a cabo la Encuesta Nacional de Nutrición (ENN), los datos sobre consumo dietético y sobre las prevalencias de deficiencias en la dieta de mujeres en edad reproductiva provenían de estudios sin representatividad en el ámbito nacional. ${ }^{20-23}$

La ENN obtuvo información sobre el estado nutrimental (antropometría y hemoglobina) y la dieta de mujeres de 12 a 49 años y niños menores de cinco años. ${ }^{24}$

El objetivo del presente trabajo fue evaluar el consumo dietético de energía y nutrimentos, su adecuación, así como posibles deficiencias en la dieta de mujeres mexicanas en edad reproductiva.

\section{Material y métodos}

La ENN se llevó a cabo en una muestra probabilística con representatividad en cuatro regiones y en el ámbito nacional. Aspectos sobre el diseño de la encuesta y la estrategia de muestreo han sido descritos en otras publicaciones. ${ }^{25}$

De entre las 19276 mujeres participantes en la ENN, se obtuvo información dietética de 9449 ; de éstas, 348 mujeres quedaron fuera del análisis por datos faltantes en energía y algunos nutrimentos. Por tanto, en el presente trabajo se presentan datos de 9101 mujeres de 12 a 49 años de edad.

La información dietética se obtuvo por medio del método de recordatorio de 24 horas, ${ }^{26}$ el cual se aplicó al ama de casa o madre de familia, quien proporcionó información sobre el consumo de alimentos o de preparaciones hecho por las mujeres habitantes de la vivienda. Con el objeto de captar la variabilidad del consumo diario, se hicieron réplicas en $5 \%$ de las mujeres de la muestra. Para fines del presente trabajo no se tomó en cuenta esa información, por carecer de ella. Se desglosaron todos los ingredientes que componen cada preparación, detallando las cantidades. Mostrando a la encuestada modelos de recipientes se cuantificó lo más exactamente posible el tamaño de las porciones consumidas porlas mujeres. A partir de estos datos se calculó el consumo individual de energía y nutrimentos, utilizando un programa de cómputo diseñado especialmente para ello y las tablas de valor nutritivo de los alimentos del Instituto Nacional de la Nutrición Salvador Zubirán ${ }^{27}$ y del United States Department of Agriculture. ${ }^{28}$

La ENN obtuvo abundante información sobre características sociodemográficas de las mujeres estudiadas por medio de entrevista directa, incluyendo: edad, número de hijos, estado fisiológico (embarazo y lactancia), escolaridad, material de construcción de la vivienda, disponibilidad de agua entubada y drenaje 
en la vivienda, y posesión de enseres domésticos. Además se obtuvieron mediciones antropométricas (peso y talla) utilizando la técnica recomendada por Jordán ${ }^{29}$ y los métodos de estandarización de Habicht. ${ }^{30}$ Parte de esta información se utilizó para el presente trabajo.

\section{Variables}

Además de la ingestión energética, se estudiaron los siguientes nutrimentos: hidratos de carbono, proteínas, lípidos (grasa total, grasa saturada, grasa polinsaturada y colesterol), hierro, calcio, vitaminas A, E, B1, B2, B6, B12, C, ácido fólico y niacina.

Para evitar la inclusión de posibles valores aberrantes se eliminaron todos aquellos datos que representaban un consumo de energía y nutrimentos mayor a cinco veces las recomendaciones de ingestión dietética (RID). ${ }^{1}$ La proporción de dichos valores fluctuaba entre 0.2 y $4.1 \%$ del total de datos.

La ingestión calórica, el porcentaje de adecuación de la misma a las recomendaciones y el consumo de los nutrimentos mencionados se calcularon para el total de mujeres estudiadas y por categorías según las siguientes características: grupo de edad (12-19, 20-29, 30-39, 40-49 años); estado fisiológico (embarazo, lactancia, no embarazo-no lactancia); condiciones de vivienda (terciles bajo, medio y alto); región (norte, centro, sur y Distrito Federal -D.F.-); área de residencia (urbana o rural), y tipo de población predominante en el municipio de residencia (indígena o no indígena).

Con información sobre características de la vivienda (material del piso, fuente de agua entubada, tipo de drenaje) y posesión de enseres domésticos (radio, televisor, refrigerador, teléfono y automóvil) se construyó un indicador de condiciones de vivienda utilizando análisis factorial de componentes principales. ${ }^{31}$ Este método ha sido utilizado en otros análisis de la ENN por los autores. ${ }^{25}$ Se seleccionaron las variables con un factor de carga cercano a 0.40 o superior. Dicho indicador fue posteriormente categorizado en terciles.

En cuanto al área de residencia de las mujeres estudiadas, la ENN clasificó los municipios como predominantemente urbanos, cuando más de la mitad de sus pobladores vivía en localidades con más de 15000 habitantes, o como predominantemente rurales, cuando más de la mitad de sus pobladores vivía en comunidades con menos de 15000 habitantes. Asimismo, los municipios fueron clasificados como predominantemente indígenas cuando al menos $40 \%$ de la población hablaba alguna lengua indígena; en caso contrario el municipio se clasificó como predominantemente no indígena.

Las regiones quedaron conformadas de la siguiente manera: norte, que comprende las entidades de Baja California, Baja California Sur, Chihuahua, Coahuila, Durango, Nuevo León, Sonora y Tamaulipas; centro, con los estados de Aguascalientes, Colima, Guanajuato, Jalisco, México (excepto los municipios conurbados del D.F.), Michoacán, Morelos, Nayarit, Querétaro, San Luis Potosí, Sinaloa y Zacatecas, y sur, que incluye Campeche, Chiapas, Guerrero, Hidalgo, Oaxaca, Puebla, Quintana Roo, Tabasco, Tlaxcala, Veracruz y Yucatán. La región D.F. quedó constituida por dicha entidad y los municipios conurbados.

Para evitar posibles valores aberrantes en las medidas antropométricas se eliminaron los datos de talla por debajo de -5 y por arriba de +4 desviaciones estándar (DE) del patrón de referencia de talla para cada año de edad ${ }^{32}$ obtenido a partir de la Hispanic Health and Nutrition Examination Survey (HHANES). Asimismo se eliminaron valores de peso para edad eíndice de masa corporal (peso en $\mathrm{kg} /$ talla en $\mathrm{m}^{2}$ ) por abajo de -3 o por arriba de +3 DE del patrón de referencia mencionado. $\square$ Los valoes eliminados por este motivo no superaron $0.2 \%$ del total de las mujeres estudiadas.

\section{A nálisis}

Se obtuvieron estadísticas descriptivas para la energía y cada uno de los nutrimentos. Todos los análisis se realizaron tomando en cuenta el peso relativo asignado a cada una de las observaciones para respetar el esquema de muestreo utilizado y conservar la representatividad del estudio. Lo anterior permite extrapolar los resultados a cerca de 10 millones de mujeres mexicanas, según el X Censo Nacional de Población y Vivienda. ${ }^{33}$

Se practicaron pruebas de análisis de varianza (ANOVA) para evaluar diferencias en las medias de ingestión de energía y nutrimentos según categorías de determinadas características estudiadas, y se usó la prueba de chi cuadrada para evaluar diferencias entre variables categóricas. ${ }^{34}$ Se eligió un nivel de significancia estadística $<0.01$.

Para conocer la proporción de mujeres con ingestiones dietéticas menores a las recomendadas se generaron variables dicotómicas para la ingestión de cada uno de los nutrimentos (excepto hidratos de carbono y lípidos) utilizando como punto de corte $50 \%$ de las RID ${ }^{1}$ según edad y estado fisiológico. Los consumos por debajo de estos puntos de corte se consideran, para fines del presente trabajo, como "probablemente 
deficientes". Dicho punto de corte corresponde aproximadamente a -2 DE de la distribución de las recomendaciones, ${ }^{2}$ y refleja muy posiblemente ingestiones inadecuadas. Asimismo, se evaluó el porcentaje de adecuación de la ingestión de energía a las recomendaciones, de acuerdo con la edad, el peso y el estado fisiológico de cada mujer, considerando una actividad física ligera (trabajo de casa, cuidado de niños, trabajo y deportes no pesados) en todos los casos. ${ }^{1}$

Se calculó el aporte calórico proporcional de proteínas, hidratos de carbono, grasas totales, grasas saturadas y polinsaturadas. Para el análisis estadístico se utilizaron los paquetes Stata $^{35}$ y SAS. ${ }^{36}$

\section{Resultados}

Los casos eliminados del análisis no fueron estadísticamente diferentes de los analizados respecto a edad, estado fisiológico, área de residencia urbana o rural e índice de masa corporal. Sin embargo, hubo diferencias entre ambos grupos en cuanto a región y predominancia indígena en el municipio de residencia $(p<0.01)$; entre el grupo excluido hubo una proporción mayor de mujeres en la región sur y en áreas con una población indígena mayor a $40 \%$.

Las características generales de las mujeres en estudio se describen en el cuadro I. Se presentan medias y desviaciones estándar para las variables continuas, y porcentajes ponderados para las variables categóricas. La media de la edad de las mujeres fue de 27.9 años. La media del peso fue de $53.8 \mathrm{~kg}$, y de la talla, $152.8 \mathrm{~cm}$, con un índice de masa corporal promedio de 22.9. La mayoría de las mujeres (casi 86\%) no estaban embarazadas ni amamantando al momento de la encuesta. El porcentaje de embarazadas fue de $5.6 \%$, y de madres amamantando (nodrizas), de $8.6 \%$. El número promedio de hijos por mujer fue de 3.6 (incluyendo nulíparas). El 58\% de las mujeres tenía una escolaridad máxima a nivel primaria.

La distribución de terciles de condiciones de vivienda se observa desigual (no exactamente $33 \%$ en cada categoría, como cabría esperar), debido posiblemente al efecto de la ponderación de las observaciones.

En cuanto a la región, aproximadamente un tercio de las mujeres correspondía al centro; otro tanto, al sur; una quinta parte, a la región norte, y una proporción similar, al D.F.

Aproximadamente una quinta parte de las mujeres residía en áreas rurales, y 6\%, en municipios con un porcentaje de población indígena mayor o igual a $40 \%$.

El cuadro II presenta los promedios, desviaciones estándar y medianas de consumo de energía y macro-

\section{Cuadro I \\ Características de la población estudiada* Encuesta Nacional de Nutrición 1988, México}

\begin{tabular}{cc}
$\begin{array}{c}\text { Edad (años) } \\
12-19\end{array}$ & 25.6 \\
\hline $20-29$ & 33.4 \\
\hline $30-39$ & 26.5 \\
\hline $40-49$ & 14.5
\end{tabular}

Estado fisiológico

Embarazada $\quad 5.7$

$\begin{array}{lr}\text { N odriza } & 5.6 \\ \text { N } 0 \text { embarazada-no nodriza } & 85.8\end{array}$

Escolaridad

\begin{tabular}{ll} 
<Primaria & 58.0 \\
\hline > Primaria & 42.0
\end{tabular}

Tercil de condiciones de vivienda

\begin{tabular}{ll} 
Bajo & 36.1 \\
\hline Medio & 33.1 \\
\hline Alto & 30.8
\end{tabular}

Región

\begin{tabular}{ll} 
Norte & 18.4 \\
\hline Centro & 29.8 \\
\hline Sur & 31.4 \\
\hline D.F. & 20.3
\end{tabular}

Area

$\begin{array}{ll}\text { Rural } & 19.2\end{array}$

Urbana $\quad 80.8$

$\%$ de población indígena

\begin{tabular}{rr}
$\geq 40 \%$ & 6.0 \\
\hline$<40 \%$ & 94.0
\end{tabular}

Media (DE)

Peso $(\mathrm{kg})$

$53.77(11.53)$

\begin{tabular}{lcl}
\hline Talla $(\mathrm{cm})$ & 152.8 & $(6.9)$ \\
\hline Indice de masa corporal $\left(\mathrm{kg} / \mathrm{m}^{2}\right)$ & 22.93 & $(4.98)$
\end{tabular}

Número de hijos ${ }^{\ddagger}$

$3.6 \quad(2.69)$

* Medias y desviación estándar o porcentajes ponderados

₹ Incluye nulíparas

nutrimentos. Las últimas fueron incluidas debido a que la mayoría de los nutrimentos estudiados, así como la energía, no presentaban una distribución normal. 
En el cuadro III se presenta el aporte calórico proporcional proveniente de proteínas, hidratos de carbono y grasas. En éste y en el cuadro anterior se presenta la información para el total de la muestra y para las diversas categorías de las variables estudiadas.
El consumo promedio de energía fue de 1721 $\mathrm{kcal} /$ día, de las cuales $15 \%$ provenía de proteínas, $60 \%$ de hidratos de carbono y $25 \%$ de grasas. Se registraron diferencias significativas en el consumo de energía entre grupos de edad, pues las mujeres más

\section{Cuadro II} Consumo diario promedio de energía, proteínas, hidratos de carbono y lípidos según características
De la población estudiada.* Encuesta Nacional de Nutrición 1988, México

Características Calorías (kcal) Proteínas $(\mathrm{g}) \quad$ Hidratos de carbono $(\mathrm{g}) \quad$ Grasas $(\mathrm{g}) \quad$ Colesterol (mg)

\begin{tabular}{|c|c|c|c|c|c|c|}
\hline \multicolumn{7}{|l|}{ Edad } \\
\hline $12-19$ & $1670(790)^{\mathrm{a}} 1510$ & $59.88(32.66)^{\mathrm{a}} 53.26$ & $253.19(140.31)$ & 222.92 & $43.58(24.82)^{\mathrm{a}} \quad 39.35$ & $268.90(233.48)^{\mathrm{a}} 200.00$ \\
\hline $20-29$ & $1745(801)^{b} 1594$ & $64.49(34.69)^{b} 57.09$ & $258.85(137.37)$ & 230.45 & $46.11(25.72)^{b} 41.50$ & $295.89(238.44)^{b} \quad 249.35$ \\
\hline $30-39$ & $1759(828)^{\text {ab }} 1603$ & $65.36(35.06)^{b} 57.91$ & $259.05(138.32)$ & 230.19 & $46.23(25.75)^{b} \quad 42.21$ & $285.09(238.35)^{\text {ab }} 233.66$ \\
\hline $40-49$ & $1684(829)^{\text {ab }} 1512$ & $61.42(34.13)^{\mathrm{ab}} 54.69$ & $253.42(144.02)$ & 222.82 & $43.45(24.93)^{\text {ab }} 40.93$ & $276.75(233.49)^{\text {ab }} 213.12$ \\
\hline \multicolumn{7}{|l|}{ Estado fisiológico } \\
\hline Embarazada & $1768(879)^{\text {ab }} 1609$ & $65.04(39.92) 56.00$ & $259.58(133.27)$ & 239.33 & $45.70(26.86) \quad 41.16$ & $300.60(245.94) \quad 242.15$ \\
\hline Nodriza & $1846(891)^{\mathrm{a}} 1680$ & $62.38(32.03) 55.55$ & $301.11(162.85)^{\mathrm{a}}$ & 268.28 & $39.75(24.02)^{\mathrm{a}} 35.97$ & $304.14(241.28) \quad 267.81$ \\
\hline No embarazada-no nodriza & $1705(796)^{b} 1553$ & $63.05(34.08) 56.12$ & $252.02(136.40)$ & 223.89 & $45.61(25.40) \quad 41.71$ & $280.81(235.64)$ \\
\hline
\end{tabular}

Escolaridad

$\begin{array}{lllllllllll}\leq \text { Primaria } & 1738(839)^{\mathrm{a}} & 1581 & 61.02(33.53)^{\mathrm{a}} & 54.43 & 270.41(145.59)^{\mathrm{a}} & 242.44 & 41.70(25.04)^{\mathrm{a}} & 37.20 & 272.88(232.40)^{\mathrm{a}} & 219.37\end{array}$

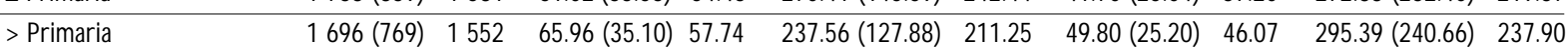

Tercil condiciones de vivienda

\begin{tabular}{|c|c|c|c|c|c|c|c|c|}
\hline Bajo & $1748(852)^{\mathrm{a}} 1585$ & $59.33(32.75)^{\text {a }} 52.40$ & $281.73(154.22)^{a}$ & 251.41 & $39.34(24.47)^{\mathrm{a}}$ & 34.68 & $268.32(229.33)$ & 218.14 \\
\hline Medio & $1721(816)^{\text {ab }} 1558$ & $63.13(35.09)^{b} 55.58$ & $254.16(135.63)^{b}$ & 228.31 & $45.75(25.67)^{b}$ & 41.55 & $279.29(238.37)$ & \\
\hline Alto & $1682(749)^{b} 1559$ & $67.63(35.00) \times 60.69$ & $228.22(118.12)^{c}$ & 204.72 & $51.22(24.92)^{c}$ & 47.89 & 1.17 & \\
\hline
\end{tabular}

Región

$\begin{array}{lllllllllll}\text { Norte } & 1700(761) & 1575 & 62.72(33.77) & 55.68 & 247.38(130.31)^{\mathrm{a}} & 223.90 & 47.74(25.51)^{\mathrm{a}} & 42.86 & 314.13(231.85) & 298.09\end{array}$

\begin{tabular}{llllllllll}
\hline Centro & $1713(784)$ & 1568 & $62.09(33.77)$ & 55.07 & $258.96(135.93)^{\text {ab }} 230.11$ & $43.95(25.61)$ & 39.79 & $252.19(231.92)^{\text {a }}$ & 170.18
\end{tabular}

\begin{tabular}{lllllllllll}
\hline Sur & $1750(847)$ & 1580 & $60.94(32.47)$ & 54.85 & $269.19(150.86)^{b}$ & 236.89 & $43.56(25.35)$ & 39.03 & $272.26(224.77)^{\mathrm{a}}$ & 227.73 \\
\hline
\end{tabular}

\begin{tabular}{lllllllllll}
\hline D.F. & $1707(832)$ & 1536 & $68.35(37.55)^{\mathrm{a}}$ & 60.47 & $242.23(131.67)^{\text {ac }}$ & 213.41 & $46.81(24.86)$ & 43.52 & $312.97(255.89)$ & 239.01
\end{tabular}

Area

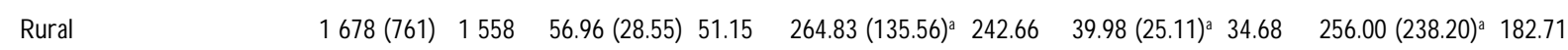

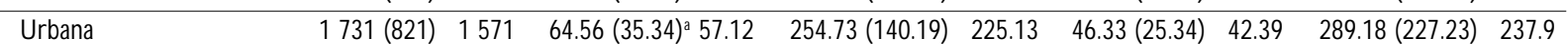

$\%$ población indígena

\begin{tabular}{llllllllllll}
$\geq 40 \%$ & $1920(797)^{\mathrm{a}}$ & 1787 & $63.85(35.40)$ & 56.13 & $316.18(180.98)^{\mathrm{a}}$ & 282.76 & $39.01(23.11)^{\mathrm{a}}$ & 34.52 & 296.93 & $(246.77)^{\mathrm{a}}$ & 225.27 \\
\hline
\end{tabular}

$\begin{array}{lllllllllll}<40 \% & 1708(797) & 1563 & 63.05(34.20) & 56.07 & 252.89(135.43) & 225.30 & 45.49(25.51) & 41.49 & 282.87(236.19) & 259.12\end{array}$

Total $\quad \begin{array}{llllllllll}1721(810) & 1568 & 63.10(34.27) & 56.07 & 256.67(139.36) & 227.54 & 45.10(25.42) & 41.15 & 283.50(236.70) & 228.34\end{array}$

* Medias y desviaciones estándar ponderadas y medianas

abc Los grupos con supraíndices distintos son significativamente diferentes entre sí $(p<0.01)$ 


\section{Cuadro III \\ Porcentaje de la ingesta calórica diaria proveniente de proteínas, Carbohidratos y grasas según características de la población estudiada. Encuesta Nacional de Nutrición 1988, México}

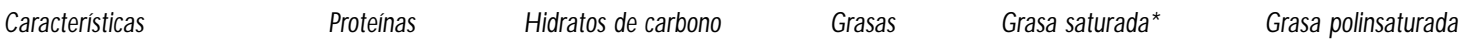

Edad

\begin{tabular}{lllllll}
$12-19$ & 14.79 & 60.56 & 24.97 & 5.39 & 4.74 & 5.03 \\
\hline $20-29$ & 15.16 & 59.57 & 25.38 & 5.48 & 5.59 & 4.89 \\
\hline $30-39$ & 15.22 & 59.52 & 25.45 & 5.59 & 4.78
\end{tabular}

Estado fisiológico

\begin{tabular}{llllll} 
Embarazada & $14.72^{\text {ab }}$ & 60.54 & 24.78 & 5.46 & 5.40 \\
\hline Nodriza & $13.94^{\mathrm{a}}$ & $65.29^{\mathrm{a}}$ & $20.84^{\mathrm{a}}$ & $4.51^{\mathrm{a}}$ & 4.82 \\
\hline No embarazada-no nodriza & $15.22^{\mathrm{b}}$ & 59.32 & 25.70 & 5.59 & 4.86
\end{tabular}

Escolaridad

\begin{tabular}{cccccc}
$\leq$ Primaria & $14.47^{\mathrm{a}}$ & $62.66^{\mathrm{a}}$ & $23.03^{\mathrm{a}}$ & $4.90^{\mathrm{a}}$ & $4.70^{\mathrm{a}}$ \\
\hline$>$ Primaria & 15.94 & 56.11 & 28.27 & 6.27 & 5.12
\end{tabular}

Tercil de condiciones de vivienda

\begin{tabular}{llllll} 
Bajo & $13.94^{\mathrm{a}}$ & $64.61^{\mathrm{a}}$ & $21.65^{\mathrm{a}}$ & $4.43^{\mathrm{a}}$ & 4.80 \\
\hline Medio & $15.09^{\mathrm{b}}$ & $59.66^{\mathrm{b}}$ & $25.48^{\mathrm{b}}$ & $5.52^{\mathrm{b}}$ & 4.88 \\
\hline Alto & $16.48^{\mathrm{c}}$ & $54.57^{\mathrm{c}}$ & $29.19^{\mathrm{c}}$ & $6.65^{\mathrm{c}}$ & 4.96
\end{tabular}

Región

\begin{tabular}{llllll} 
Norte & $15.12^{\mathrm{a}}$ & $58.27^{\mathrm{a}}$ & $26.96^{\mathrm{a}}$ & $5.93^{\mathrm{a}}$ & $4.87^{\mathrm{a}}$ \\
\hline Centro & $14.80^{\mathrm{ab}}$ & $60.77^{\mathrm{b}}$ & $24.44^{\mathrm{b}}$ & $5.47^{\mathrm{b}}$ & $4.27^{\mathrm{b}}$ \\
\hline Sur & $14.42^{\mathrm{b}}$ & $61.48^{\mathrm{c}}$ & $24.06^{\mathrm{b}}$ & $4.70^{\mathrm{c}}$ & $5.37^{\mathrm{c}}$ \\
\hline D.F. & $16.50^{\mathrm{c}}$ & $57.64^{\mathrm{a}}$ & $26.61^{\mathrm{a}}$ & $6.31^{\mathrm{a}}$ & $5.06^{\mathrm{ac}}$
\end{tabular}

Area

\begin{tabular}{llllll} 
Rural & $14.09^{\mathrm{a}}$ & $63.17^{\mathrm{a}}$ & $22.68^{\mathrm{a}}$ & $5.02^{\mathrm{a}}$ & 4.71 \\
\hline Urbana & 15.32 & 59.12 & 25.83 & 5.60 & 4.93
\end{tabular}

$\%$ población indígena

\begin{tabular}{rlllll}
$\geq 40 \%$ & $13.64^{\mathrm{a}}$ & $65.89^{\mathrm{a}}$ & $20.33^{\mathrm{a}}$ & $3.84^{\mathrm{a}}$ & 4.95 \\
\hline$<40 \%$ & 15.17 & 59.52 & 25.54 & 5.59 & 4.88 \\
Total & 15.08 & 59.90 & 25.22 & 5.50 & 4.89
\end{tabular}

* No incluye colesterol

abc Los grupos con supraíndices distintos son significativamente diferentes entre sí $(p<0.01)$

jóvenes tenían el menor consumo en términos absolutos. Las mujeres de 20 a 29 y de 30 a 39 años fueron las que consumieron mayor cantidad de proteínas, carbohidratos y lípidos, lo que las diferenciaba significativamente de las demás.

De acuerdo con el estado fisiológico, las mujeres nodrizas fueron las que tuvieron mayor consumo de energía en comparación con el resto de mujeres, con un consumo significativamente menor de grasas y mayor de hidratos de carbono, de los cuales proviene, en promedio, más de $65 \%$ de su ingestión calórica.

Las mujeres con menor grado de escolaridad presentaron consumos inferiores de proteínas, grasas y colesterol, así como un mayor consumo de calorías 
y de hidratos de carbono, de tal modo que obtuvieron una mayor proporción de energía derivada de éstos en comparación con las mujeres con un nivel de escolaridad más alto.

Las mujeres con el nivel más bajo de condiciones de vivienda consumieron mayor cantidad de energía respecto a las categorías media y alta, así como mayor cantidad de hidratos de carbono. Por otro lado, las mujeres con mejores condiciones de vivienda consumieron una mayor cantidad y proporción de proteínas y lípidos.

En cuanto al lugar de residencia, las mujeres de la región sur consumieron significativamente una mayor cantidad de calorías e hidratos de carbono. En la región correspondiente al Distrito Federal y áreas conurbadas se presentó un mayor consumo de proteínas, mientras que el mayor consumo de grasas se registró en el norte del país. El mayor consumo de colesterol se presentó en las regiones norte y D.F.

No hubo diferencias significativas en el consumo de energía entre áreas urbanas y rurales; sin embargo, la ingestión de proteínas y lípidos fue significativamente mayor en las zonas urbanas. En las áreas rurales se presentó un mayor consumo de hidratos de carbono, al igual que en las zonas predominantemente indígenas, donde el consumo calórico fue superior al observado en zonas no indígenas.

Los cuadros IV y V ilustran la adecuación del consumo de energía, proteínas y micronutrimentos según las recomendaciones diarias y de acuerdo con las características de la población estudiada.

La media de adecuación a la recomendación de consumo de energía indica que las mujeres consumen en promedio $84.6 \%$ de las calorías que deberían consumir.

Las mujeres más jóvenes mostraron una mejor adecuación, mientras que las mayores presentaron la más baja. Las embarazadas y nodrizas presentaron un porcentaje de adecuación más bajo en relación con el resto de las mujeres.

Respecto a los micronutrimentos, los porcentajes más altos de ingestión inadecuada se observaron para la vitamina B6 (75\%), la vitamina A $(70 \%)$, el folato $(69 \%)$ y la vitamina C $(56 \%)$.

En las mujeres con menor escolaridad se observaron mayores proporciones de ingestiones inadecuadas de proteína y de todos los micronutrimentos, exceptuando el hierro, en comparación con las mujeres con mayor nivel de escolaridad, de tal modo que las diferencias resultaron estadísticamente significativas.

Las mujeres en el tercil bajo de condiciones de vivienda presentaron el mayor porcentaje de ingestión inadecuada para proteína y para todos los micronu- trimentos, exceptuando el hierro. En este último caso el mayor porcentaje de inadecuación se observó entre las mujeres del tercil alto.

En la región sur se encontró un mayor porcentaje de mujeres con ingestión inadecuada de proteína y micronutrimentos, excepto para niacina, vitamina $\mathrm{E}$ y hierro. En el D.F. se observó una situación opuesta, pues se presentó el menor porcentaje de mujeres con consumos inferiores a los recomendados.

Respecto al área de residencia (rural o urbana), no se observaron diferencias significativas en el porcentaje de mujeres con un consumo energético menor a los requerimientos diarios. Sin embargo, los porcentajes con un bajo consumo proteico y de micronutrimentos fueron significativamente mayores en el área rural, excepto en el caso de la vitamina B1 y el hierro. Lo mismo puede decirse de las mujeres habitantes de áreas predominantemente indígenas, aunque no mostraron diferencias en cuanto al consumo de calcio en comparación con las demás.

\section{Discusión}

Las proporciones de calorías provenientes de proteínas, carbohidratos y grasas (alrededor de 15, 60 y 25\%, respectivamente) indican que la dieta promedio fue equilibrada en composición de macronutrimentos.

Es interesante observar que las mujeres provenientes del área rural, de la región sur, de zonas con gran proporción de población indígena y cuyas condiciones de vivienda fueron clasificadas en el tercil más bajo, presentaron un mejor porcentaje de adecuación de la ingestión energética en relación con las recomendaciones. Sin embargo, entre las mujeres de estos grupos se obtuvieron los porcentajes más altos de dietas probablemente deficientes en cuanto a proteína y micronutrimentos, así como un mayor consumo de hidratos de carbono. Lo contrario sucede con el hierro, para el que dichas mujeres presentaron menores porcentajes de consumo inadecuado. Las diferencias observadas en cuanto a macronutrimentos sugieren que las fuentes de hierro en estos grupos son principalmente de origen vegetal. El hierro derivado de estos alimentos tiene una menor biodisponibilidad que el proveniente de los alimentos de origen animal. Aun cuando la dieta cubre en gran medida las necesidades energéticas de las mujeres mexicanas pertenecientes a los grupos más desposeídos, la calidad de ésta en términos de proteína y micronutrimentos es insatisfactoria, posiblemente debido a la poca variedad que existe en su composición. ${ }^{37}$

Es de hacer notar que la ENN fue diseñada para tener representatividad únicamente en los planos re- 


\section{Cuadro IV \\ Porcentaje de adecuación del consumo calórico a las recomendaciones diarias y porcentajes DE MUJERES MEXICANAS EN EDAD REPRODUCTIVA CON UN CONSUMO DIARIO DE PROTÉ́NAS Y MINERALES INFERIOR a la mitad de las recomendaciones. Encuesta Nacional de Nutrición 1988, México}

\begin{tabular}{|c|c|c|c|c|}
\hline \multirow[b]{2}{*}{ Características } & \multirow{2}{*}{$\begin{array}{c}\text { \% de adecuación } \\
\text { de calorías* }\end{array}$} & \multicolumn{3}{|c|}{ Porcentaje de mujeres con consumos inferiores a $50 \%$ de las RID } \\
\hline & & Proteínas & Calcio & Hierro \\
\hline \multicolumn{5}{|l|}{ Edad } \\
\hline $12-19$ & $93.35^{\mathrm{a}}$ & 5.64 & $45.35^{\mathrm{a}}$ & 23.12 \\
\hline $20-29$ & 82.23 & 8.00 & $34.03^{b}$ & 22.30 \\
\hline $30-39$ & 81.87 & 7.27 & $24.43^{c}$ & 21.15 \\
\hline $40-49$ & 79.69 & 8.21 & $24.35^{c}$ & 21.40 \\
\hline
\end{tabular}

\begin{tabular}{lcccc}
$\begin{array}{l}\text { Estado fisiológico } \\
\text { Embarazada }\end{array}$ & 75.35 & 14.21 & 40.90 & $64.45^{\mathrm{a}}$ \\
\hline Nodriza & 74.89 & 14.36 & 41.43 & $13.58^{\mathrm{b}}$ \\
\hline No embarazada-no nodriza & $86.20^{\mathrm{a}}$ & $6.05^{\mathrm{a}}$ & $31.62^{\mathrm{a}}$ & $20.12^{\mathrm{c}}$
\end{tabular}

\begin{tabular}{lllll}
$\begin{array}{l}\text { Escolaridad } \\
\leq \text { Primaria }\end{array}$ & 84.82 & $8.79^{\mathrm{a}}$ & $34.23^{\mathrm{a}}$ & $19.66^{\mathrm{a}}$ \\
\hline$>$ Primaria & 84.28 & 5.15 & 31.28 & 25.34
\end{tabular}

Tercil

\begin{tabular}{lllll} 
Bajo & $86.73^{\mathrm{a}}$ & $10.00^{\mathrm{a}}$ & $37.72^{\mathrm{a}}$ & 19.77 \\
\hline Medio & $84.17^{\mathrm{a}}$ & $6.66^{\mathrm{b}}$ & $32.63^{\mathrm{b}}$ & 21.89 \\
\hline Alto & $81.96^{\mathrm{b}}$ & $4.62^{\mathrm{c}}$ & $28.02^{\mathrm{c}}$ & $25.04^{\mathrm{a}}$
\end{tabular}

\begin{tabular}{lllll}
$\begin{array}{l}\text { Región } \\
\text { N orte }\end{array}$ & $81.70^{\mathrm{a}}$ & 6.90 & 35.41 & $26.80^{\mathrm{a}}$ \\
\hline Centro & $84.71^{\mathrm{ab}}$ & 6.06 & $27.00^{\mathrm{a}}$ & 20.18 \\
\hline Sur & $87.30^{\mathrm{b}}$ & $9.50^{\mathrm{a}}$ & 39.31 & 21.24 \\
\hline D.F. & $82.91^{\mathrm{a}}$ & 5.71 & $29.76^{\mathrm{a}}$ & 21.82
\end{tabular}

\begin{tabular}{lcccc}
$\begin{array}{l}\text { Area } \\
\text { Rural }\end{array}$ & 82.74 & $9.43^{\mathrm{a}}$ & $35.63^{\mathrm{a}}$ & 2.36 \\
\hline Urbana & 85.05 & 6.71 & & 22.52 \\
$\begin{array}{c}\text { Indígena } \\
\geq 40 \%\end{array}$ & & & 32.50 & 17.84 \\
\hline$<40 \%$ & $94.34^{\mathrm{a}}$ & $11.14^{\mathrm{a}}$ & 33.02 & 22.34 \\
Total & 83.98 & 6.98 & 32.99 & 22.07
\end{tabular}

* Las recomendaciones diarias fueron calculadas según las ecuaciones para estimar el gasto energético en reposo con base en la edad y el peso, multiplicándose el resultado por un factor de actividad ligera

abc Los grupos con supraíndices distintos son significativamente diferentes entre sí $(p<0.01)$

gional y nacional, y que no es representativa para algunos de los niveles de desagregación mencionados en el presente trabajo, tales como: área urbana o rural, o bien, predominantemente no indígena o indígena, y escolaridad, por mencionar algunos. Por lo tanto, la in- formación correspondiente a estos estratos deberá tomarse como una aproximación y con la debida reserva.

El consumo energético diario promedio observado en el presente estudio fue similar al de mujeres mexicanas residentes en EUA, según datos de la HHANES. ${ }^{38}$ 


\section{Cuadro V}

Porcentaje de mujeres mexicanas en edad Reproductiva con un consumo de vitaminas menor a la mitad de las recomendaciones diarias. Encuesta Nacional de Nutrición 1988, México

Características

B1 $\quad$ B2 $\quad$ B6

B12

A

Folato

Niacina

C

$\mathrm{E}$

Edad

\begin{tabular}{llllllllll}
$12-19$ & 9.31 & 30.88 & 75.56 & 34.88 & 71.39 & 67.09 & $42.87^{\mathrm{a}}$ & 54.85 & 46.00 \\
\hline $20-29$ & 9.39 & 28.99 & 74.16 & 31.40 & 69.68 & 70.99 & $38.63^{\mathrm{ab}}$ & 56.87 & 44.88 \\
\hline $30-39$ & 8.21 & 26.53 & 75.65 & 31.88 & 67.89 & 69.01 & $36.45^{\mathrm{b}}$ & 54.67 & 45.24 \\
\hline $40-49$ & 9.89 & 28.79 & 74.73 & 32.74 & 72.88 & 68.22 & $39.68^{\text {ab }}$ & 58.68 & 45.08
\end{tabular}

Estado fisiológico

\begin{tabular}{lccccccccc} 
Embarazada & $19.81^{\mathrm{a}}$ & $36.94^{\mathrm{a}}$ & 82.79 & 31.56 & 64.82 & 89.39 & $45.05^{\mathrm{a}}$ & 59.05 & 44.50 \\
\hline Nodriza & $13.87^{\mathrm{b}}$ & $55.15^{\mathrm{b}}$ & 87.40 & $43.21^{\mathrm{a}}$ & $85.03^{\mathrm{a}}$ & 88.10 & $56.30^{\mathrm{b}}$ & $75.49^{\mathrm{a}}$ & 48.19 \\
\hline No embarazada nodriza & $7.95^{\mathrm{c}}$ & $25.58^{\mathrm{c}}$ & $73.35^{\mathrm{a}}$ & 31.77 & 68.95 & $65.96^{\mathrm{a}}$ & $37.20^{\mathrm{c}}$ & 53.96 & 45.07
\end{tabular}

Escolaridad

\begin{tabular}{cccccccccc} 
< Primaria & $9.40^{\mathrm{a}}$ & $35.94^{\mathrm{a}}$ & $81.18^{\mathrm{a}}$ & $38.56^{\mathrm{a}}$ & $77.37^{\mathrm{a}}$ & $76.24^{\mathrm{a}}$ & $44.22^{\mathrm{a}}$ & $64.05^{\mathrm{a}}$ & $48.45^{\mathrm{a}}$ \\
\hline$>$ Primaria & 8.85 & 18.96 & 66.90 & 25.93 & 60.07 & 59.76 & 32.47 & 45.25 & 45.33
\end{tabular}

Tercil

\begin{tabular}{llllllllll} 
Bajo & 9.46 & $41.59^{\mathrm{a}}$ & $84.87^{\mathrm{a}}$ & $44.57^{\mathrm{a}}$ & $81.97^{\mathrm{a}}$ & $79.54^{\mathrm{a}}$ & $46.05^{\mathrm{a}}$ & $67.14^{\mathrm{a}}$ & 48.80 \\
\hline Medio & 8.48 & $26.98^{\mathrm{b}}$ & $75.46^{\mathrm{b}}$ & $33.25^{\mathrm{b}}$ & $69.55^{\mathrm{b}}$ & $70.24^{\mathrm{b}}$ & $40.63^{\mathrm{b}}$ & $56.45^{\mathrm{b}}$ & 46.32 \\
\hline Alto & 9.19 & $14.95^{\mathrm{c}}$ & $63.62^{\mathrm{c}}$ & $21.24^{\mathrm{c}}$ & $56.05^{\mathrm{c}}$ & $55.84^{\mathrm{c}}$ & $29.31^{\mathrm{c}}$ & $42.38^{\mathrm{c}}$ & $40.42^{\mathrm{a}}$
\end{tabular}

Región

\begin{tabular}{lrllllllll} 
N orte & $10.86^{\mathrm{a}}$ & $24.57^{\mathrm{a}}$ & $73.10^{\mathrm{a}}$ & $28.10^{\mathrm{a}}$ & $68.32^{\mathrm{a}}$ & $64.82^{\mathrm{a}}$ & 39.61 & 58.52 & 42.06 \\
\hline Centro & $6.95^{\mathrm{b}}$ & $27.93^{\mathrm{a}}$ & $79.48^{\mathrm{b}}$ & $33.85^{\mathrm{b}}$ & $72.33^{\mathrm{b}}$ & $74.44^{\mathrm{b}}$ & 43.21 & 61.21 & $53.01^{\mathrm{a}}$ \\
\hline Sur & $11.20^{\mathrm{a}}$ & $39.89^{\mathrm{b}}$ & $80.04^{\mathrm{b}}$ & $42.22^{\mathrm{c}}$ & $76.62^{\mathrm{b}}$ & $77.38^{\mathrm{b}}$ & 41.88 & 60.56 & 42.28 \\
\hline D.F. & $7.54^{\mathrm{b}}$ & $16.26^{\mathrm{c}}$ & $63.03^{\mathrm{c}}$ & $22.39^{\mathrm{d}}$ & $58.13^{\mathrm{c}}$ & $52.92^{\mathrm{c}}$ & $29.09^{\mathrm{a}}$ & $39.04^{\mathrm{a}}$ & 41.50
\end{tabular}

Area

\begin{tabular}{llllllllll} 
Rural & 9.48 & $40.25^{\mathrm{a}}$ & $82.41^{\mathrm{a}}$ & $43.24^{\mathrm{a}}$ & $76.42^{\mathrm{a}}$ & $81.28^{\mathrm{a}}$ & $44.83^{\mathrm{a}}$ & $63.37^{\mathrm{a}}$ & $52.10^{\mathrm{a}}$ \\
\hline Urbana & 9.05 & 26.04 & 73.33 & 30.49 & 68.60 & 66.28 & 37.97 & 54.33 & 43.73
\end{tabular}

$\%$ indígena

\begin{tabular}{rlllllllll}
$\geq 40 \%$ & 9.66 & $44.03^{\mathrm{a}}$ & $84.06^{\mathrm{a}}$ & $45.27^{\mathrm{a}}$ & $84.50^{\mathrm{a}}$ & $79.55^{\mathrm{a}}$ & $45.04^{\mathrm{a}}$ & $70.75^{\mathrm{a}}$ & $39.87^{\mathrm{a}}$ \\
\hline$<40 \%$ & 9.10 & 27.80 & 74.48 & 31.98 & 69.17 & 68.45 & 38.92 & 55.16 & 45.61 \\
& & & & & & & & & \\
Total & 9.13 & 28.78 & 74.99 & 32.58 & 70.10 & 69.07 & 39.29 & 56.04 & 45.29
\end{tabular}

abc Los grupos con supraíndices distintos son significativamente diferentes entre sí $(p<0.01)$

En contraste, los consumos absolutos de proteína $(63.1$ vs $74.3 \mathrm{~g}$ ), grasas totales (45.1 vs $68.6 \mathrm{~g}$ ) y colesterol (283.5 vs $352.5 \mathrm{mg}$ ) fueron menores entre las participantes de la ENN, pues presentaron un mayor consumo de hidratos de carbono (256.7 vs $205.2 \mathrm{~g}$ ) y un mayor porcentaje de energía proveniente de éstos (60 vs 48\%). En relación con el consumo diario prome- dio de micronutrimentos y en comparación con las mujeres de la HHANES, las participantes de la ENN tuvieron un consumo cuatro veces menor de vitamina A, tres veces menor de ácido fólico, dos veces menor de vitamina $C y$ un consumo similar de vitamina $\mathrm{E}$, calcio y hierro. Esta comparación pretende únicamente dar una idea de las similitudes existentes entre los hallaz- 
gos informados y otras encuestas poblacionales, por lo que no se ajustó la ingestión de nutrimentos por otros factores.

Los resultados sobre ingestión deficiente de vitaminas son consistentes con la revisión realizada por Rosado y colaboradores ${ }^{20,21}$ sobre estudios llevados a cabo en México. Destacan en este particular las ingestiones bajas de retinol, vitamina $C$, niacina y riboflavina, aunque en el presente estudio también se observaron deficiencias dietéticas importantes en vitamina B6 y ácido fólico. En cuanto al consumo observado de niacina es necesario mencionar que no se añadió a éste la sexagésima parte del triptófano que se transforma en niacina, por no contar con esta información en la base de datos.

El método de recordatorio de 24 horas usado en la presente investigación ha sido empleado en otros estudios dietéticos de poblaciones. Aunque este método recoge información de un solo día y, por tanto, no capta la variabilidad en el consumo diario de alimentos, la información proporcionada en una muestra tan grande como la estudiada permite obtener una buena aproximación respecto de los niveles de consumo. Además, su uso posibilita la comparación de resultados con otros estudios que utilizan el mismo método. ${ }^{39} \mathrm{El}$ recordatorio de 24 horas puede tener sesgos debido a fallas en la memoria de los individuos. En general, se ha observado que el método tiene una tendencia a subestimar entre 10 y $25 \%$ el consumo de diferentes nutrimentos, en comparación con el peso directo de alimentos. Dicha subestimación es mayor para algunos nutrimentos como calcio, proteína animal y hierro, y menor para el consumo de energía. ${ }^{40} \mathrm{~A}$ pesar de ello, estas diferencias no invalidan las comparaciones entre las categorías estudiadas. Con todo y estas limitaciones, la magnitud de algunas deficiencias en la dieta de las mujeres estudiadas es notable; tal es el caso de las vitaminas A, B6, C y ácido fólico.

Por otro lado, los casos eliminados del análisis no difirieron notoriamente respecto a los demás, salvo que en los primeros existió una mayor proporción de individuos en la región sur y en áreas con predominio de población indígena. Lo anterior tendría como consecuencia una subrepresentación de mujeres en los estratos mencionados, donde se observaron mayores deficiencias en la dieta; por lo tanto, muy probablemente los resultados subestiman la magnitud de algunas de ellas. A pesar de las limitaciones mencionadas, los resultados que aquí se presentan indican deficiencias importantes en la dieta, las cuales afectan mayormente a las mujeres embarazadas y a las nodrizas, a las de menor grado de escolaridad y menor nivel socioeconómico, así como a aquellas residentes en áreas predominantemente rurales e indígenas y a las que habitan en la región sur del país. Es por ello que los programas y los recursos destinados a mejorar la calidad de la dieta de las mujeres mexicanas en edad reproductiva (por ejemplo, mediante suplementación, fortificación de alimentos, alimentación complementaria, educación alimentaria y monitoreo del estado nutricional) deben dirigirse especialmente a estos grupos.

\section{Referencias}

1. N ational Research Council. Recommended dietary allowances. 10a. edición. W ashington, D.C.: N ational A cademy Press, 1989.

2.W ada L, King JC. Trace element nutrition during pregnancy. $C$ lin 0 bstet Gynecol 1994;37(3):574-586.

3. Langer A, Romero M. El embarazo, el parto y el puerperio. ¿Bajo qué condiciones se reproducen las mujeres en México? En: Langer A, Tolbert K, ed. Mujer: sexualidad y salud reproductiva en México. México, D.F.: EDAMEX, 1996:13-37.

4. Lara M, Acevedo M. Incorporación de la mujer al trabajo remunerado: repercusiones para su salud reproductiva. En: Langer $A$, Tolbert $K$, ed. Mujer: sexualidad y salud reproductiva en México. México, DF: EDAMEX, 1996:119-151.

5. Krasovec $K$, Anderson MA, ed. Maternal nutrition and pregnancy outcomes. Anthropometric assessment. Scientific Publication núm. 529. W ashington, D.C.: Pan A merican Health O rganization, 1991.

6. Johnson AA, Knight EM, Edwards $\mathrm{CH}, 0$ yemade UJ, Jackson $\mathrm{O}$, W estrey $E$ et al. Dietary intakes, anthropometric measurements and pregnancy outcomes. J N utr 1994;124:936S-942S.

7. Susser M. Maternal weight gain, infant birth weight, and diet: Causal sequences. Am J C lin N utr 1991;53:1384-1396.

8. Keen $C L$, Zidenberg-Cherr S. Should vitamin-mineral supplements be recommended for all women with childbearing potential? A m J C lin N utr 1994;59suppl:532-539.

9. Kramer M. Determinants of low birth weight: Methodological assessment and meta-analysis. Bull World Health Organization 1987;65(5): 663-737.

10. Prentice AM, Cole TJ, Foord FA, Lamb W H,W hitehead RG. Increased birthweight after prenatal dietary supplementation of rural $A$ frican women. Am J Clin N utr 1987;46:912-925.

11.Viteri F.The consecuences of iron deficiency and anemia in pregnancy. En:Allen L, Kung J, Lönnerdal B, ed. N utrient regulation during pregnancy, lactation and infant growth.Advances in experimental medicine and biology. N ueva York: Plenum Press, 1994:127-139.

12. Scholl T, Hediger M, Fischer $R$, Shearer J. Anemia vs iron deficiency: Increased risk of preterm delivery in a prospective study. Am J Clin N utr 1992;55:985-988.

13. Henly SJ,Anderson CM, A very MD, Hills-Bonczyk SG, Potter S, D uckelt $L J$. Anemia and insufficient milk in first-time mothers. Birth 1995;22(2):87-92.

14. Long K, Rivera J, Rivera M, Hernández-Avila M, Lezana M. Feeding patterns of Mexican infants recorded in the $1988 \mathrm{~N}$ ational $N$ utrition Survey. Salud Publica Mex 1995;37(2):120-129.

15. N eel N R, Alvarez J0. Chronic fetal malnutrition and vitamin A in cord serum. Eur J Clin N utr 1990;44(3):207-212.

16. G hebremeskel K, Burns L, Burden TJ, H arbige L, Costeloe K, Powell JJ et al. Vitamin A and related essential nutrients in cord blood: Relation- 
ships with anthropometric measurements at birth. Early Hum Dev 1994;39:177-188.

17. Shah R, Rajalakshmi R.Vitamin A status of the newborn in relation to gestational age, body weight and maternal nutrition status. Am J Clin N utr 1984;40:794-800.

18. $N$ avarro J, Bourgeay M, D esquilbet $N$, Herve F, Lallemand D. The vitamin status of low birth weight infants and their mothers.J Pediatr G astroenterol N utr 1984;3:744-748.

19. C zeizel A. Prevention of congenital abnormalities by periconceptional multivitamin supplementation. BMJ 1993;306:1645-1648.

20. Rosado JL, Bourges H, Saint-Martin B. D eficiencia de vitaminas y minerales en México. Una revisión crítica del estado de la información. I. Deficiencia de minerales. Salud Publica Mex 1995;37(2):130-139.

21. Rosado JL, Bourges H, Saint-Martin B. D eficiencia de vitaminas y minerales en México. Una revisión crítica del estado de la información. II. Deficiencia de vitaminas. Salud Publica Mex 1995;37(5):452-461.

22. Chávez A, ed. Encuestas nutricionales en México. División de Nutrición del Instituto Nacional de Nutrición Salvador Zubirán, México, D.F.: 1963.

23. Madrigal H, Chávez A, Moreno-Terrazas O, García T, Gutiérrez G. Consumo de alimentos y estado nutricional de la población del medio rural mexicano. Rev Invest Clin 1986;38 suppl.: 9-19.

24. Sepúlveda J. Estado nutricional de los preescolares y las mujeres en México: resultados de una encuesta nacional probabilística. México, D.F.: Academia $\mathrm{N}$ acional de Medicina, México, D.F.:1989.

25. Rivera J, González-C ossío T, Flores M, Hernández-A vila M, Lezana MA, Sepúlveda J. Déficit de talla y emaciación en menores de cinco años en distintas regiones y estratos en México. Salud Publica Mex 1995;37(2): 95-107.

26. Gibson RS. Principles of N utrition Assessment. Food consuption of individuals. N ueva York: 0 xford University Press, 1990:37-47.

27. Hernández M, Chávez A, Bourges H.Valor nutritivo de los alimentos mexicanos. Tablas de uso práctico. México, D.F.: Instituto $\mathrm{N}$ acional de la Nutrición, 1977.
28. United States Department of Agriculture. Food composition tables handbook.W ashington D.C.:C onsumer \& Food Economics Institute, Agricultural Research Service, 1984.

29. Jordán JR. Desarrollo humano en Cuba. La Habana: Editorial Científico-Técnica,1979.

30. Habicht JP. Estandarización de métodos epidemiológicos cuantitativos sobre el terreno. Bol 0 ficina Sanit Panam 1974;76:375-385.

31. Kleinbaum D G, Kupper LL, Muller KE.Applied regression analysis and other multivariate methods. 2a. edición. Boston: PW S-Kent Publishing, 1988.

32. $N$ ational $C$ enter for Health Statistics. Anthropometric data and prevalence of overweight for Hispanics: 1982-84. Maryland: N CHS, 1989. DHHS Publication N 0. (PHS) 89-1689.

33. Consejo Nacional de Población. X Censo Nacional de Población y Vivienda. México, D.F.: CO N APO, 1980.

34. Pagano M, G auvreau K. Principles of biostatistics. D uxbury Press, 1993. 35. Stata Corporation. Stata Reference Manual: Release 3.1. 6a. edición Ed. College Station, TX. 1993.

36. SAS Institute Inc. SAS/STAT U ser's Guide,Version 6. 4a. edición Cary, NC. 1989.

37. O PS-ILSI. C onocimientos actuales sobre nutrición. 6a. edición. Publicación Científica núm. 532. W ashington, D.C.: O PS-ILSI, 1991.

38. Guendelman $\mathrm{S}, \mathrm{Abrams} B$. D ietary intake among Mexican-A merican women: Generational differences and a comparison with white non-Hispanic women. Am J Public Health 1995;85:20-25.

39. Creed H. Una clasificación de métodos para la evaluación del consumo dietético y prácticas de alimentación. En: Madrigal H, Martínez $\mathrm{H}$, ed. Manual de encuestas de dieta. Serie Perspectivas en Salud Pública. Cuernavaca, México: Instituto N acional de Salud Pública,1996:51-61. 40. Parra S, Romieu I, Hernández-Avila M, Madrigal H. Usos y limitaciones de los métodos de encuesta dietética. En: Madrigal $H$, Martínez $H$. Manual de encuestas de dieta. Serie Perspectivas en Salud Pública. Cuernavaca, México: Instituto N acional de Salud Pública, 1996:25-50. 